\title{
Protein synthesis factors (RF1, RF2, RF3, RRF, and tmRNA) and peptidyl-tRNA hydrolase rescue stalled ribosomes at sense codons.
}

\author{
Serafín Vivanco-Domínguez \\ Centro de Investigación y de Estudios Avanzados del I. P. N. \\ José Bueno-Martínez \\ Centro de Investigación y de Estudios Avanzados del I. P. N. \\ Gloria León-Avila \\ Escuela Nacional de Ciencias Biológicas del I. P. N.

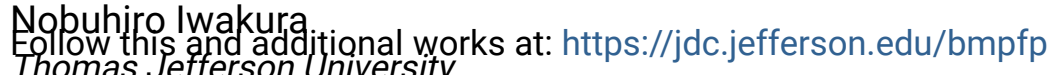 \\ Thomas Jefferson University \\ Part of the Medical Biochemistry Commons, and the Medical Molecular Biology Commons \\ Akira kaii knowy how access to this document benefits you
}

Recommended Citation

See next page for additional authors

Vivanco-Domínguez, Serafín; Bueno-Martínez, José; León-Avila, Gloria; Iwakura, Nobuhiro; Kaji, Akira; Kaji, Hideko; and Guarneros, Gabriel, "Protein synthesis factors (RF1, RF2, RF3, RRF, and tmRNA) and peptidyl-tRNA hydrolase rescue stalled ribosomes at sense codons." (2012).

Department of Biochemistry and Molecular Biology Faculty Papers. Paper 38.

https://jdc.jefferson.edu/bmpfp/38

This Article is brought to you for free and open access by the Jefferson Digital Commons. The Jefferson Digital Commons is a service of Thomas Jefferson University's Center for Teaching and Learning (CTL). The Commons is a showcase for Jefferson books and journals, peer-reviewed scholarly publications, unique historical collections from the University archives, and teaching tools. The Jefferson Digital Commons allows researchers and interested readers anywhere in the world to learn about and keep up to date with Jefferson scholarship. This article has been accepted for inclusion in Department of Biochemistry and Molecular Biology Faculty Papers by an authorized administrator of the Jefferson Digital Commons. For more information, please contact:

JeffersonDigitalCommons@jefferson.edu. 


\section{Authors}

Serafín Vivanco-Domínguez, José Bueno-Martínez, Gloria León-Avila, Nobuhiro Iwakura, Akira Kaji, Hideko Kaji, and Gabriel Guarneros 


\title{
As submitted to:
}

\section{Journal of Molecular Biology}

And later published as:

\section{Protein synthesis factors (RF1, 2, 3, RRF, and tmRNA) and peptidyl- tRNA hydrolase rescue stalled ribosomes at sense codons}

\author{
Volume 417, Issue 5, 13 April 2012, Pages 425-439
}

DOI: $10.1016 / j . j m b .2012 .02 .008$

Serafin Vivanco-Domínguez ${ }^{\mathrm{a}}$, José Bueno-Martínez ${ }^{\mathrm{a}}$, Gloria León-Avilab ${ }^{\mathrm{b}}$, Nobuhiro Iwakurac ${ }^{\mathrm{c}}$, Akira Kaji ${ }^{\mathrm{d}}$, Hideko Kaji ${ }^{\mathrm{c}, 1}$, and Gabriel Guarneros ${ }^{\mathrm{a}, 1}$

${ }^{a}$ Depto. de Genética y Biología Molecular, Centro de Investigación y de Estudios Avanzados del I. P. N., P.O. Box 14-740, Mexico City, 07000; 'Depto. de Zoología, Escuela Nacional de Ciencias Biológicas del I. P. N., Carpio y Plan de Ayala, Mexico City, 11340; 'Dept. of Biochemistry and Molecular Biology, Kimmel Cancer Center, Thomas Jefferson University, 1020 Locust Street, Philadelphia, PA 19107; and dDept. of Microbiology, School of Medicine, University of Pennsylvania, 3610 Hamilton Walk, Philadelphia, PA 19104 
Author contributions: S.V.-D., J.B.-M., A.K., H.K., N.I., and G.G. designed the research; S.V.-D., J.B.-M., and G.L.-A. were responsible for conducting the experiments; N.I. confirmed some of the experimental results; S.V.-D., and J.B.-M. contributed equally to this work; S.V.-D., G.L.-A., N.I., A.K., H.K., and G.G. critically analyzed data and wrote the paper.

The authors declare no conflict of interest.

Running title: Factors involved in the rescue of stalled ribosomes

Keywords: Release factors, RF1, RF2, RF3; Ribosome recycling factor, RRF; Transfer-messenger RNA, tmRNA; Peptidyl-tRNA hydrolase, Pth; Rescue of stalled ribosomes.

${ }^{1}$ To whom correspondence may be addressed.

E-mail: hideko.kaji@jefferson.edu or gguarner@cinvestav.mx

\section{ABSTRACT}

During translation, ribosomes stall on mRNA when the aminoacyl-tRNA to be read is not readily available. The stalled ribosomes are deleterious to the cell and should be rescued to maintain its viability. To investigate the contribution of some of the cellular translation factors on ribosome rescuing, we provoked stalling at AGA codons in mutants which affected the factors, and then analyzed the accumulation of oligopeptidyl- (peptides of up to 6 amino acid residues, oligopep-) tRNA or polypeptidyl(peptides of more than 300 amino acids in length, polypep-) tRNA associated with ribosomes. Stalling was achieved by starvation for aminoacyl-tRNA ${ }^{A r g} 4$ upon induced expression of engineered lacZ ( $\beta$-galactosidase $\underline{Z}$ ) reporter gene harboring contiguous 
AGA codons close to the initiation codon or at internal codon positions together with minigene AUGAGAUAA accompanied by reduced peptidyl-tRNA hydrolase. Our results showed accumulations of peptidyl-tRNA associated with ribosomes in mutants for release factors (RF1, RF2, and RF3), ribosome recycling factor (RRF), peptidyl-thNA

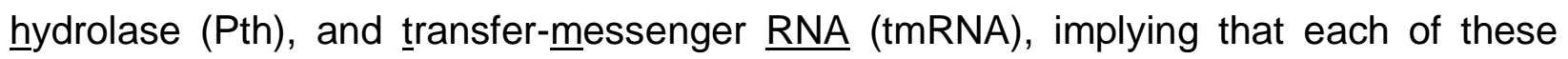
factors cooperate in rescuing stalled ribosomes. The role of these factors in ribosome releasing from the stalled complex may vary depending on the length of the peptide in the peptidyl-tRNA. RF3 and RRF rescue stalled ribosomes by "drop off" of peptidyltRNA, while RF1, RF2 (in the absence of termination codon), or Pth may rescue by hydrolyzing the associated peptidyl-tRNA. This is followed by the disassembly of the ribosomal complex of tRNA and mRNA by RRF and EF-G (elongation factor $\underline{\mathrm{G}}$ ).

\section{INTRODUCTION}

A round of protein synthesis ends when the ribosome reaches a stop codon (UAG, UGA, or UAA) in the mRNA. In bacteria, release factors (RF1 or RF2) recognize the stop codons to induce hydrolysis of peptidyl-tRNA (pep-tRNA) at the ribosomal peptidyl transferase center (PTC) and release the complete peptide. ${ }^{1-6}$ RF3/GTP accelerates the dissociation of RF1 and RF2 from ribosome ${ }^{7,8}$ leaving a post-termination complex (PoTC), which consists of mRNA, tRNA and ribosome. PoTC is disassembled by RRF and EF-G/GTP and its components are used in new rounds of protein synthesis. ${ }^{9-14}$

Factors mentioned above are not limited to disassembly of PoTC but they must play a role also in handling stalled ribosomes at sense codons to ensure high efficiency 
of the ribosome usage. There are non-programmed and programmed ribosome stalling. The latter functions as a mechanism of gene regulation. ${ }^{15-25}$ Non-programmed ribosomal stalling occurs in two different manners. First, it can occur with ribosome stalled at the end of non-stop mRNA. It has been reported that tmRNA but not RF1, RF3, or RRF are involved in the rescue of stalled ribosomes in this case. ${ }^{26}$ Furthermore, YaeJ protein, which carries a GGQ motif (like RFs), induces the hydrolysis of pep-tRNA that resides in a ribosome stalled on a non-stop mRNA. ${ }^{27,28}$ In addition, ArfA protein also rescues stalled ribosomes from non-stop mRNAs. ${ }^{29}$ Second, the stalling occurs when aminoacyl-tRNA is depleted, leading to empty A-site. The empty A-site has "hungry codon" because it does not have aminoacyl-tRNA corresponding to the triplet. The hungry codon affects cell's growth. ${ }^{30}$ These stalled ribosomes are rescued by a mechanism where mRNA is cleaved by RelE (ribonuclease which cuts mRNA at the Asite) followed by the action of tmRNA. Rel stands for "relax" meaning that the control of RNA synthesis by amino acid is relaxed in the relE mutant as opposed to the wild type which "stringently" controls RNA synthesis by amino acid availability. Alanyl-tmRNA enters the A-site and accepts the nascent peptide group of the pep-tRNA in the Psite. ${ }^{31-36}$ In addition, RF3 or RRF/EF-G, ${ }^{37-39}$ or RRF/EF-G/IF3 "drop off" pep-tRNA ${ }^{40}$ and these factors collaborate in vivo. ${ }^{41}$

Pep-tRNA released from ribosomes is hydrolyzed by Pth (peptidyl-tRNA hydrolase). ${ }^{42,43}$ In Pth reduced cells, expression of minigene coding for two amino acids cause the release of dipep-tRNA from ribosomes decreasing the aminoacyl-tRNA pool. ${ }^{44,45}$ Lambda phage codes for short ORF and produces short oligopeptidyl-tRNA, which has to be hydrolyzed by Pth for the growth of the phage. In the rap mutant, the 
level of Pth is very much reduced and hence the lambda phage cannot grow in this strain. Rap stands for "Restriction of $\underline{\mathrm{At}^{+}} \underline{\text { Phage". }} \mathrm{Att}^{+}$phage means the phage with the gene related to phage attaching to the host. $\mathrm{Att}^{+}$contains the DNA region which codes for the minigene. The rap mutation restricts the phage growth because with low Pth activity, the host cannot stand the large amount of minigene product (oligopep-tRNA) made by the phage. This mutation has no detectable effect on bacterial growth. ${ }^{46}$ In our past publications, non-programmed ribosome stalling was created by the use of $p t h(r a p)$ together with minigene (AUGAGAUAA) resulting in the shortage of tRNA ${ }^{\operatorname{Arg} 4.47}$

In this paper, we asked whether or not the factors which mediate translation termination, PoTC disassembly, and hydrolysis of pep-tRNA were involved in rescuing stalled ribosomes caused by the lack of sufficient aminoacyl-tRNA or release factors corresponding to the A-site codon. We either removed or reduced the in vivo activity of the factors and estimated the accumulation of pep-tRNA in the cell as an index of ribosome stalling. Our evidence suggests that all six factors (RRF, RF1, RF2, RF3, Pth, and tmRNA) collaborate to rescue stalled ribosomes with hungry codon at the A-site in E. coli. We propose that RF1 and RF2 promote in vivo hydrolysis of the ester bond of pep-tRNA despite that A-site does not have the termination codon in stalled ribosomes. Furthermore, Pth appears to cleave oligopep-tRNA on the ribosome helping to disassemble the stalled ribosomes with the oligopep-tRNA.

\section{MATERIALS AND METHODS}

\section{Bacterial Strains, Plasmids, and Growth Conditions}


The bacterial strains and plasmids used in this study are listed in Table 1. P1 phagemediated transduction was used to transfer genetic markers. The bacterial constructs were selected by resistance to antibiotics or temperature sensitivity. All strains used were derivative of P90C (Table 1). P90C has the following characteristics: $\Delta$ (gpt-lac): gtp (guanine-xanthine phosphoribosyltransferase) and lac genes (lacA, $Y, Z$, and I) located between $5.5 \mathrm{~min}$ and $7.8-7.9 \mathrm{~min}$ are deleted. Also pro $A$ and proB at $5.6 \mathrm{~min}$ (proA; y-glutamyl phosphate reductase and proB; y-glutamyl kinase) are missing. The lac deletion was necessary to induce the lac gene in the plasmids used in this paper. Each of the strains with one or two plasmids were grown in LB broth (Figs. 1, 2, 3, 6a and $6 \mathrm{~b})$ or M9 minimal medium supplemented with $20 \mu \mathrm{g} / \mathrm{ml}$ of each amino acid, $20 \mu \mathrm{M}$ biotin and $20 \mu \mathrm{M}$ thiamine (Figs. 4b, 5, and 6c). When required, $100 \mu \mathrm{g} / \mathrm{ml}$ ampicillin (Amp), $30 \mu \mathrm{g} / \mathrm{ml}$ kanamycin (Kan), $50 \mu \mathrm{g} / \mathrm{ml}$ chloramphenicol $(\mathrm{Cm})$ or $12.5 \mu \mathrm{g} / \mathrm{ml}$ tetracycline (Tet) were added to the medium. After cultures reached sufficient optical density, minigene and/or lacZ genes expression were induced with $1 \mathrm{mM}$ isopropylthiogalactoside (IPTG).

\section{Extraction and analysis of total oligopep-tRNA ${ }^{\text {Arg4 }}$}

Oligopep-tRNAs (Figs. 1, 2, 3, 6a, and 6b) were extracted in acidic conditions as described previously ${ }^{48,49}$ with some modifications. After induction, $5 \mathrm{ml}$ of LB cultures were immediately mixed with an equal volume of acid phenol. The aqueous phase was recovered by centrifugation at $4^{\circ} \mathrm{C}$ and extracted with acid phenol (repeated two times). Aqueous phase was treated with $\mathrm{EtOH}$ at $-20^{\circ} \mathrm{C}$. The precipitate present in the RNA 
pellet was washed with $70 \% \mathrm{EtOH}$, and was treated with diethyl pyrocarbonate (DEPC). Aminoacyl-tRNA in the extract was hydrolyzed with $10 \mathrm{mM} \mathrm{CuSO}_{4}$, for $30 \mathrm{~min}$ at $37^{\circ} \mathrm{C}$. Oligopep-tRNA remains intact under these conditions. ${ }^{50}$ The extracted RNA was electrophoresed on urea-acrylamide (6\%) acid gels, ${ }^{51}$ and blotted onto Hybond $\mathrm{N}^{+}$ Nylon membranes (Amersham). $\operatorname{tRNA}^{\mathrm{Arg} 4}$ was detected by Northern blot with a specific $\left.{ }^{32} \mathrm{P}\right]$-oligonucleotide probe for tRNA ${ }^{\text {Arg4 }}$ (Table 2).

\section{Oligopep-tRNA ${ }^{\mathrm{Arg} 4}$ and $\mathrm{mRNA}$ in ribosome fractions}

One hundred $\mathrm{ml}$ LB cultures $\left(0.3 \mathrm{OD}_{600}\right)$ (Fig. 2) were induced as described in the legend to Fig.1. Cells were suspended in buffer containing $50 \mu \mathrm{g} / \mathrm{ml} \mathrm{Tet},{ }^{41,51}$ disrupted in a French press (450 psi), and centrifuged at $30,000 \times g$ at $4^{\circ} \mathrm{C}$ for 30 min to obtain S-30. S-30 was incubated in the presence (+) or absence (-) of $2.5 \mathrm{mM}$ puromycin for $10 \mathrm{~min}$ at $37^{\circ} \mathrm{C}$, and was centrifuged at $100,000 \times g$ at $4^{\circ} \mathrm{C}$ for $2 \mathrm{hrs}$ to obtain $\mathrm{S}-100$ (S) and ribosomal $(\mathrm{R})$ fractions. S-100 was concentrated by precipitation with $\mathrm{EtOH}$ at $-20^{\circ} \mathrm{C}$. RNA obtained from S-100 and ribosomal pellets by the acid phenol method were treated with $\mathrm{CuSO}_{4}$ as described in the previous section. Three $\mu$ of each fraction were resolved in acidic polyacrylamide gel (Fig. 2a) or in $2 \%$ formaldehyde agarose gel $^{52}$ (Fig. 2b). Pep-tRNA ${ }^{\mathrm{Arg} 4}$ and lacZ AGA3-6 mRNA were probed using specific $\left[{ }^{32} \mathrm{P}\right]-$ oligonucleotides (Table 2).

$\beta$-gal-polypep-tRNA ${ }^{\text {Leu1 }}$ detection. 
In Fig. 4, $5 \mathrm{ml}$ of cultures were grown at 28 or $32^{\circ} \mathrm{C}\left(0.5-0.6 \mathrm{OD}_{600}\right)$, induced as described in the figure legend, boiled in the Laemmli buffer, ${ }^{53}$ and subjected to $10 \%$ SDS-PAGE. The N-terminal His-6 tagged $\beta$-gal polypeptides were detected by Western blot using anti-His-6 antibody. In Fig. 5, $100 \mathrm{ml}$ of cultures were induced as in Fig. 4 (32/42), and were processed as described ${ }^{54}$ with some modifications. The lysozymetreated cell pellet was suspended in $450 \mu$ of modified lysis buffer $(20 \mathrm{mM}$ Hepes-KOH $\mathrm{pH}$ 7.8, $50 \mathrm{mM} \mathrm{MgCl}_{2}, 50 \mathrm{mM} \mathrm{NH} \mathrm{Cl}_{4}, 1 \mathrm{mM}$ DTT, $200 \mathrm{U}$ DNAse I, $100 \mu \mathrm{g} / \mathrm{ml} \mathrm{Cm}$ ), and the cells were disrupted by adding $25 \mu \mathrm{l}$ of $5 \%$ Brij58 and sonicated. The lysate was spun down at $30,000 \times g$ for $20 \mathrm{~min}$ at $4^{\circ} \mathrm{C}$. The extract thus obtained was fractionated by $10-40 \%$ SDGC (sucrose density gradient centrifugation) prepared in lysis buffer without DNAse I and centrifuged in a Beckman rotor (SW40 Ti rotor) for $3 \mathrm{hrs}$ at 34,000 rpm $(145,000 \times g)$. The absorbance of each fraction $(500 \mu \mathrm{l}$, collected from the top of the gradient) was measured at $260 \mathrm{~nm}$. To analyze polypep-tRNA, each fraction $(60 \mu \mathrm{l})$ was suspended in Tris-acetate buffer and subjected to Tris-acetate SDS-PAGE is as described ${ }^{55}$. Proteins and RNAs were blotted onto nitrocellulose or nylon membrane in $25 \mathrm{mM}$ Tris- $\mathrm{HCl} \mathrm{pH} 8.3$ and $192 \mathrm{mM}$ glycine. The nitrocellulose membrane was subjected to Western blot using anti-His-6 antibody to detect His-tagged $\beta$-gal. The nylon membrane was subjected to Northern blot to detect $\beta$-gal polypep-tRNA ${ }^{\text {Leu1 }}$ by $\left[{ }^{32} \mathrm{P}\right]$-oligonucleotide specific probe for tRNA ${ }^{\text {Leu1 }}$ (Table 2).

\section{Hydrolysis of polypep-tRNA ${ }^{\text {Leu1 }}$ by Pth}


In Fig. 6c. pHZ352-353-MR expression was induced for 5 min at $32^{\circ} \mathrm{C}$ to allow sequestering of $\mathrm{TRNA}^{\mathrm{Arg} 4}$ by the AGA minigene expression. Cells were then switched to $42^{\circ} \mathrm{C}$ for $15 \mathrm{~min}$ to inactivate temperature-sensitive RF1. These cells $(20 \mathrm{ml}$ culture, 0.5 $\left.\mathrm{OD}_{600}\right)$ were suspended in $500 \mu \mathrm{l}$ of $10 \mathrm{mM} \mathrm{NaOAc} \mathrm{pH} 5.0$, and sonicated. Debris was removed by centrifugation and the solution was adjusted to $\mathrm{pH} 7.2$. Then, $50 \mu \mathrm{l}$ of the Pth reaction buffer 10X (100 mM Tris- $\mathrm{HCl} \mathrm{pH} \mathrm{7.6,} 100 \mathrm{mM} \mathrm{MgOAc}, 200 \mathrm{mM} \mathrm{NH}_{4} \mathrm{Cl}$, and $60 \mathrm{mM} \beta-\mathrm{ME}$ ) and $\mathrm{N}$-terminal-His tagged Pth were added to the extract and incubated at $37^{\circ} \mathrm{C}$ for $20 \mathrm{~min}$. His-tagged $\beta$-gal was detected as described in previous sections.

\section{RESULTS}

Reduced Pth, inactive RRF, absence of RF3 or deletion of tmRNA increases ribosome stalling

To determine the role of RRF, RF3, tmRNA or Pth in the release of pep-tRNA from in vivo stalled ribosomes, we examined the accumulation of oligopep-tRNA ${ }^{\operatorname{Arg} 4}$ in strains deficient in these factors that expressed placZ AGA3-6 construct $^{45,56}$ (Fig. 1a and Table 1). The expression of lacZ AGA3-6 mRNA was induced at $42^{\circ} \mathrm{C}$ and the total RNA was analyzed by Northern blotting (Fig. 1b). In wild type cells, no appreciable oligopeptRNA $^{\text {Arg4 }}$ was observed (Fig. 1b, lane 1). If one of the factors analyzed were to contribute to the release of oligopep-tRNA ${ }^{\mathrm{Arg} 4}$ from the stalled ribosome, we would expect that in the absence of that factor, the percentages (shown below each lane) of 
oligopep-tRNA ${ }^{\mathrm{Arg} 4}$ of total tRNA $\mathrm{Arg}^{\mathrm{Ar} 4}$ would increase. In pth(rap) strain, a mutant with reduced Pth activity, ${ }^{57,58}$ high accumulation of oligopep-tRNA ${ }^{\text {Arg4 }}$ was observed $(97.5 \%$ of total tRNA ${ }^{\text {Arg4}}$ ) (Fig. 1b, lane 2). In this mutant, the oligopep-tRNA ${ }^{\text {Arg4 }}$ might be ribosome-bound or free. In frrTs (the gene coding for factor for recycling of ribosome is mutated to produce temperature sensitive RRF, RRFTs ${ }^{59}$ ) accumulation of oligopeptRNA $^{\text {Arg4 }}$ increased at the non-permissive temperature (1.9 vs. 95.5\%; Fig. 1b, lanes 1 and 3). frrTs is the original LJ14 mutant of frr and the colony counts at $32^{\circ} \mathrm{C}$ was the same as the wild type but it was less than $10^{-5}$ of wild type at $42^{\circ} \mathrm{C}$. Bacterial growth rate of this mutant was slightly impaired even at $32^{\circ} \mathrm{C}$ while it was identical to that of wild type at $28^{\circ} \mathrm{C}$ (unpublished data). In mutant $\Delta p r f C$ (the gene for peptide chain release factor $\underline{\mathrm{C}}$ or RF3 is deleted, $\Delta \mathrm{RF} 3$ ), oligopep-tRNA ${ }^{\mathrm{Arg}} 4$ accumulation increased compare to wild type cells ( $58.1 \%$ vs. $1.9 \%$; lanes 4 and 1 ) supporting the idea that these factors help "drop off" of pep-tRNA from ribosomes. ${ }^{37-39}$ In the absence of tmRNA ( $\Delta s s r A$ strain, the strain missing the gene coding for small stable $\underline{R} N A \underline{A}$ ), a molecule that rescues stalled ribosomes, ${ }^{31}$ oligopep-tRNA ${ }^{\text {Arg4 }}$ increased compared to that in wild type cells (lanes 5 vs. 1 ) indicating that oligopep-tRNA ${ }^{\text {Arg4 }}$ was on stalled ribosomes. In all mutant strains, the accumulation of oligopep-tRNA ${ }^{\operatorname{Arg} 4}$ caused by the shortage of tRNA ${ }^{\operatorname{Arg} 4}$ was prevented by over-expression of tRNA ${ }^{\text {Arg } 4}$ from the pDC952 construct under the same experimental conditions as those of lanes 1-5 (Fig. 1b, lanes 6-10). This observation that extra tRNA ${ }^{\text {Arg4 }}$ relieves stalled ribosomes under similar conditions has been reported. ${ }^{60}$ 


\section{The oligopep-tRNA ${ }^{\text {Arg4 }}$ detected with reduced factors is bound to the ribosomal P- site and protects the mRNA}

In the preceding section, we observed that the oligopep-tRNA ${ }^{\mathrm{Arg} 4}$ accumulated despite the presence of wild type Pth in some cases (Fig. 1b, lanes 3 to 5). These data imply that the oligopep-tRNA ${ }^{A r g} 4$ is bound to the ribosome because ribosome protects, to some extent, the bound pep-tRNA ${ }^{\text {Arg } 4}$ from Pth. ${ }^{42}$

To confirm this view, the crude extracts of cells with induced lacZ AGA 3-6 mRNA expression were treated, with or without puromycin, an antibiotic which accepts peptidyl group from the oligopep-tRNA ${ }^{\mathrm{Arg} 4}$ bound at the ribosomal P-site. The presence of oligopep-tRNA ${ }^{\mathrm{Arg} 4}$ was analyzed by Northern blot in both supernatant (S) and ribosomal fractions (R) (Fig. 2a).

In strains with frrTs (RRFTs), deletion of the gene for RF3 ( $\triangle \mathrm{RF} 3$ ), or deletion of the gene for tmRNA ( $\triangle \mathrm{tmRNA}$ ), oligopep-tRNA ${ }^{\mathrm{Arg} 4}$ was detected only in the ribosomal fraction because of the presence of wild type Pth, which hydrolyzes free pep-tRNA very efficiently. Treatment with puromycin resulted in the removal of the oligopep-tRNA ${ }^{\text {Arg4 }}$ from the ribosome in the form of oligopeptidyl puromycin. This shows that it was bound to the P-site in a physiologically active, ${ }^{61}$ stalled complex (Fig. $2 \mathrm{~A}$, panels $3-6$ ). In the strain with reduced Pth activity (Pth(rap)), oligopep-tRNA ${ }^{\text {Arg4 }}$ was found in both ribosomal and supernatant fractions (panel 3). The oligopep-tRNA ${ }^{\text {Arg4 }}$ found in the supernatant in panel 3 must have been released from ribosomes by RRF and RF3 which are present in Pth(rap) cells. It is important to note that oligopep-tRNA ${ }^{\operatorname{Arg} 4}$ was found on the ribosome when each of four factors including Pth were reduced or deleted. 
This implies that, Pth, like other factors, plays an important role in the rescue of the stalled ribosome.

Because the ribosome prevents mRNA hydrolysis by cellular ribonucleases, ${ }^{62,63}$ we examined if the ribosomal stalling provoked by depletion of tRNA ${ }^{\text {Arg } 4}$ protected a fragment of lacZ AGA3-6 mRNA containing the AGA3-6 sequence. The RNA samples used in Fig. 2a were probed with oligonucleotides specific for lacZ AGA3-6 mRNA (Fig. $2 \mathrm{~b}$, Table 2). The result showed that the 5 '-end fragment of mRNA was detected in ribosomal fractions of the mutant strains pth(rap), frrTs, or with deletion of the gene for RF3. They all accumulated oligopep-tRNA ${ }^{\text {Arg } 4}$ (compare Fig. $2 a$ and b). The band must be lacZ AGA3-6 mRNA because it was not detected in the absence of IPTG (Fig. $2 b$ panel 2). The amount of 5'-end lacZ AGA3-6 mRNA accumulated was higher in all mutants than in wild type cell (Fig. 2b compare panels 1 and 3-6).

\section{Stalled ribosomes with minigene and Met-Arg-tRNA ${ }^{\operatorname{Arg} 4}$ appear when protein synthesis factors are missing or reduced but no effect of deletion of tmRNA}

The results in Figs. 1 and 2 show that each one of RF3, RRF, Pth and tmRNA removes oligopep-tRNA ${ }^{A r g} 4$ from stalled ribosomes containing a sense codon in the A-site. We asked whether these factors process the oligopep-tRNA on stalled ribosomes that contain a stop codon in the A-site. To answer this question, a minigene construct carrying the ORF sequence ATGAGAUAA (Fig. 3a) was expressed in mutants of Pth, RF3, RRF or tmRNA. The levels of Met-Arg-tRNA ${ }^{\operatorname{Arg} 4}$ and tRNA ${ }^{\text {Arg4 }}$ were analyzed. The

results shown in Fig. $3 b$ revealed that in wild type cells no Met-Arg-tRNA ${ }^{\mathrm{Arg} 4}$ was found 
(lane 1). In contrast, with reduced amount of Pth (pth(rap)), all of tRNA ${ }^{\operatorname{Arg} 4}$ is in Met-ArgtRNA $^{\text {Arg4 }}$ (lane 2). This is due to two reasons. First, Met-Arg-tRNA ${ }^{\text {Arg4 }}$ released from the stalled ribosome could not be hydrolyzed. ${ }^{44}$ Second, the Pth activity to hydrolyze oligopep-tRNA on the stalled ribosome is compromised leaving bound Met-Arg-tRNA ${ }^{\text {Arg4 }}$ on the ribosome. In other mutants, Met-Arg-tRNA ${ }^{A r g 4}$ was also detected. Thus, when RRF is inactivated, the accumulated Met-Arg-tRNA ${ }^{\operatorname{Arg} 4}$ was found on the ribosome indicating ribosome stalling (Fig. $3 \mathrm{C}$ ) occurred. It should be noted in this case that ribosome-bound deacylated tRNA was found (lane 3). This shows that the PoTC remained due to the inactivation of RRF. Important exception to the effect of various factors relieving stalled ribosomes was the effect of missing tmRNA ( $\Delta s s r A$ strain). There was no effect of the loss of tmRNA (Fig. 3b, lane 5). Biological significance of this observation will be dealt with in the discussion section. The data shown in Fig. 3 indicate that minigene can also stall ribosomes suggesting that there is not enough RF1 and 2 in the cell to deal with the large amount of termination complex formed by the minigene. This would cause the empty A-site with the hungry termination codon like the case of depletion of tRNA ${ }^{\mathrm{Arg} 4}$ creating hungry AGA codon.

\section{Absence of active RRF, RF3 or tmRNA results in complexes with stalled ribosome harboring long peptide chain-tRNA (polypep-tRNA)}

We asked whether defective factors lead to polypep-tRNA accumulation on stalled ribosomes as was observed with oligopep-tRNA ${ }^{\mathrm{Arg} 4}$. To answer this question, cells with defective or missing factors were transformed with pHZ352-353-MR (lacZ AGA352-353 
mRNA $)^{64}$ (Fig. 4a and Table 1). Translation of this mRNA to the point where tandem AGAs would result in truncated $\beta$-galactosidase. As shown in Fig. $4 b$, this could be achieved upon tRNA ${ }^{A r g 4}$ depletion by the simultaneous induction of minigene ATGAGATAA and reduced level of Pth in Pth(rap) cells. In this system, there are only two AGA codons in the $\beta$-galactosidase gene. Furthermore, the position of the two tandem codon AGAs is well in the mRNA compared to the system described in Figs. 1 to 3 . This makes it more difficult to release truncated $\beta$-galactosyl-tRNA from the ribosome. Therefore, it is necessary to express the minigene together with low level of Pth for causing the shortage of tRNA ${ }^{\text {Arg4 }}$. As shown below, in this particular case, polypep-tRNA ${ }^{\text {Leu1 }}$ is accumulated due to the shortage of tRNA ${ }^{\text {Arg4 }}$ because CUG coding for Leu is located at position 351 (Fig. 4a).

As shown in Fig. 4, the Western blot assay using an anti-His-6 antibody showed the incomplete $\beta$-gal-polypeptide (arrow 2) in the double mutants $p t h(r a p) / \Delta s s r A$, pth(rap)/frrTs $\left(42^{\circ} \mathrm{C}\right)$, and pth(rap)/deletion of the gene for RF3 (Fig. 4b, lanes 4,6 , and 10). The size of the incomplete $\beta$-gal-polypeptide is similar to that obtained with a lacZ variant carrying a stop codon in position 353 (pHZ-MR construct, Table 1). Either at the permissive temperature of RRFTs $\left(28^{\circ} \mathrm{C}\right)$ or by over-expression of wild type RRF (lanes 7 and 8), the stalled ribosomes were rescued. In both cases, intermediate size $\beta$-gal bands that correspond to fragments normally produced by high rate translation of lacZ were accumulated ${ }^{65}$ (arrow 3). Induction of the plasmid expression in single mutants (Pth (pth(rap), tmRNA $(\Delta s s r A)$, RF3 $(\Delta p r f C)$ or RRF (frrTs at non permissive temperature)) produced full size $\beta$-gal protein only (Fig. 4b, arrow 1 in lanes $2,3,5$, and 9). 
The non-acid condition used for SDS-PAGE in Fig. 4 probably hydrolyzed the polypep-tRNA ${ }^{\text {Leu1 }}$ produced during the ribosomal stalling in the middle of lacZ mRNA. To avoid this problem, the crude extracts from single mutant pth(rap) and double mutants $p t h($ rap $) / f r r T s$, and $p t h(\operatorname{rap}) / p r f C$ transformed with $\mathrm{pHZ352-353-MR}$ were fractionated in SDGC (Fig. 5a) and the incomplete $\beta$-gal was detected by Western blot (Fig. 5b) under acidic conditions. In all mutants, full size $\beta$-gal (120 kDa, arrow 1) was detected. The ribosomal fraction of the single mutant, pth(rap), should carry various size $\beta$-galactosidase which distributed throughout the gel making it undetectable under the experimental conditions (panel Ab). Two incomplete $\beta$-gal-polypeptides (arrows 2 and 3) were found in the ribosomal fraction (white arrows) of the double mutants only (panels $\mathrm{Bb}$ and $\mathrm{Cb}$ ). The band labeled with arrow 2 corresponds to incomplete $\beta$-galpolypep-tRNA $A^{\text {leu1 }}$, and the other band (arrow 3) corresponds to the truncated $\beta$-galpolypeptide which lost tRNA leu1 during the analysis. This was further supported by probing with an oligonucleotide specific for tRNA ${ }^{\text {Leu1 }}$ (panels $\mathrm{Bc}$ and $\mathrm{Cc}$ ). The fast moving band (arrow 4 in Fig. 5c) corresponding to free tRNA ${ }^{\text {Leu1 }}$ was mostly located in the supernatant fractions. These data (Figs 4 and 5) suggest that RRF, RF3, and tmRNA, collaborate in rescuing stalled ribosomes that harbor polypep-tRNA ${ }^{\text {Leu1 }}$ at internal locations of mRNA when sufficient depletion of tRNA ${ }^{\text {Arg4 }}$ was achieved through pth(rap) mutation and the presence of minigene.

\section{RF1 or RF2 relieves ribosomes stalled at AGA codons regardless of the length of pep-tRNA}


It is known that nascent polypeptide goes through the $50 S$ tunnel ${ }^{66}$. This would make it difficult to release polypep-tRNA ${ }^{\text {Leu1 }}$ mentioned in the preceding section from the stalled ribosome by "drop off" of pep-tRNA. It would be easier to rescue the stalled ribosomes if the ester bond of the bound polypep-tRNA ${ }^{\text {Leu1 }}$ is cleaved. In this connection, it has recently been reported that RF2 cleaves pep-tRNA miscoded at the P-site on ribosomes with an empty A-site and sense codon. ${ }^{67}$ Their complex is similar to our stalled ribosome in that it has sense codon at the empty A-site while it is different in that the Psite of their ribosome is mismatched. Despite the difference, we wondered if RF1 or RF2 cleaves the ester bond of pep-tRNA bound to the stalled ribosome. This would result in the rescue of stalled ribosomes by the release factors without involving the "drop off" of pep-tRNA from the 50 S tunnel.

To examine this possibility, we tested if inactivation of RF1 or RF2 provokes oligopep-tRNA ${ }^{\text {Arg4 }}$ accumulation by expression of lacZ AGA3-6 mRNA in vivo. The placZ AGA3-6 mRNA was expressed in mutant prfA1 (the gene for peptide chain release factor $\underline{A}$ is mutated to code for temperature sensitive RF1, RF1Ts) or prfB2 (the gene for peptide chain release factor $\underline{B}$ is mutated to code for temperature sensitive RF2, RF2Ts). As shown in Fig. $6 a$ and $b$, at the non-permissive temperature for mutant RF1 or RF2, the induced expression of lacZ AGA3-6 mRNA was accompanied by the accumulation of oligopep-tRNA ${ }^{\operatorname{Arg} 4}$. This is consistent with the new concept that RFs rescue the stalled ribosome with sense codon at the empty A-site and matched oligopep-tRNA ${ }^{\mathrm{Arg} 4}$ at the P-site. We then examined if RF1 rescues stalled ribosomes harboring a polypep-tRNA ${ }^{\text {Leu1 }}$. In the experiment shown in Fig. $6 \mathrm{c}$, the plasmid, pHZAGA352-353-MR, was introduced into pth(rap)/prfA1 double and prfA1 single 
mutants. The accumulation of incomplete $\beta$-gal-polypep-tRNA ${ }^{\text {Leu1 }}$ and the polypeptide without tRNA ${ }^{\text {Leu1 }}$ was detected by Western blotting under conditions that preserved the ester bond of $\beta$-gal-polypep-tRNA ${ }^{\text {Leu1 }}$. The results revealed that the $\beta$-gal-polypeptRNA $^{\text {Leu1 }}$ (arrow 2) was present in extracts of the double mutant pth(rap)/prfA1Ts (lane 2 in Fig. 6c,) but not in that of the single mutant prfA1 (lane 1). In these two lanes we observed complete $\beta$-gal-polypeptide (arrow 1) representing the case where ribosome did not stall at AGA352-353. The arrow 2 band represents $\beta$-gal-polypep-tRNA ${ }^{\text {Leu1 }}$ because it was converted to incomplete $\beta$-gal-polypeptide (arrow 3 ) upon treatment of the extract with Pth (lanes 3 and 4 in Fig. 6c). We conclude that RFs are involved in rescuing stalled ribosomes by hydrolysis of pep-tRNA associated with the stalled ribosome regardless of the peptide size.

\section{DISCUSSION}

Various factors are involved in the rescue of stalled ribosomes with hungry codon at the A-site.

In this paper, we show that factors Pth, RRF, RF1, RF2, RF3, and tmRNA together contribute in the rescue of stalled ribosomes caused by empty A-site. It should be emphasized that the rescue of the ribosomes at the end of termination-less mRNA (for example ${ }^{26}$ ) is different from the cases discussed in this paper. Stalled ribosomes were created by expressing genes carrying tandem of AGA codons alone or together with expression of the minigene, AUGAGAUAA, under reduced Pth. Stalling was detected by the accumulation of pep-tRNA and mRNA on the ribosome. Since all of the factors collaborate to reduce the stalled ribosomes, missing one factor results in the 
accumulation of stalled ribosomes. We postulate three possible pathways for the disposal of the stalled ribosomes (complex A, Fig. 7). Pathway (ii) is the well accepted pathway and pathway (iii) has already been proposed for ribosomes with oligopep-tRNA 37 as well as for those with polypep-tRNA $A^{22,39,41}$ though in vitro experiments did not support the release of polypep-tRNA by RRF and EF-G. ${ }^{68}$

\section{RF1, 2 and 3 play a role in the rescue of stalled ribosomes.}

Pathway (i) shows a new concept that RF1, 2 and 3 participate in disassembling the stalled ribosome complex (Fig. 6). The empty A-site gives RF2 the opportunity to bind to the ribosome even without the termination codon on the A-site if the P-site is mismatched. ${ }^{67}$ The P-site of stalled ribosome is not mismatched but we speculated that RFs may recognize the stalled ribosomes even with matched P-site. This speculation appears to be supported as shown in Fig. 6. In fact, some matched P-site bound peptRNA has been reported to be hydrolyzed, though much less extent, by RF2. ${ }^{67}$ How RFs recognize the stalled ribosomes or ribosomes with mismatched $\mathrm{P}$-site remains unknown at the present moment. This action of RF1 and 2 is facilitated by RF3 which recycles RF1 or 2 . The fact that the loss of RF3 alone can cause ribosome stalling with polypeptRNA $^{\text {Leu1 }}$ (Figs. 4 and 5) suggests that recycling of RFs ${ }^{7,8}$ and binding ${ }^{69}$ of RF1 and RF2 stimulated by RF3, may play important roles in the rescue of stalled ribosomes. After the RFs-dependent loss of the peptidyl group that goes through the ribosomal tunnel, ${ }^{70,71}$ the rest of the complex will be readily disassembled by RRF and EF-G for recycling of the ribosome, mRNA and tRNA (Fig. 7, complexes B to E). 
The role of RRF in the rescue of stalled ribosomes with pep-tRNA.

RRF plays a direct role in pathway (iii) and the accumulation of the stalled ribosome upon inactivation of RRF through this pathway is readily understandable. In addition, we propose that depletion of RRF causes the accumulation of the stalled ribosomes through pathway (i). When RRF is inactivated, its substrate, PoTC with the termination codon at the A-site, accumulates. Since PoTC has affinity for RF1 or $2,{ }^{2}$ the accumulated PoTC can sequester the available RF1 and RF2. Thus, depletion of RRF would have the same effect as inactivating RF1 or RF2.

\section{tmRNA is not involved in the rescue of stalled ribosomes at the termination codon.}

Pathway (ii) of Fig. 7 involves tmRNA and should function for the rescue of stalled

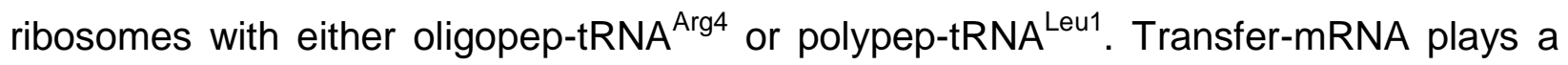
critical role, as has been proposed ${ }^{72,73}$ for rescue of stalled ribosomes. The accumulation of pep-tRNA, due to the deletion of the gene for tmRNA, reveals the role

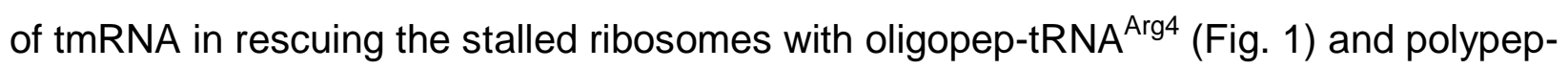
tRNA $^{\text {leu1 }}$ (Fig. 4b). One important exception in the tmRNA rescue activity was observed with ribosomes containing a stop codon in A-site (ATGAGATAA minigene, Fig. 3 lane 5). The lack of tmRNA function in this case is perhaps physiologically important because tmRNA should not interfere with the normal termination process catalyzed by RF1 or RF2. Similar failure of tmRNA to rescue stalled ribosomes with the termination codon at the A-site has been reported. ${ }^{22}$ 


\section{The role of Pth in the rescue of stalled ribosomes.}

In systems used in this paper, we postulate that Pth has two roles. First, Pth helps to replenish tRNA by hydrolyzing free oligopep-tRNA. This is the role we traditionally ascribed to Pth in our past publications dealing with stalled ribosomes. In this connection, we used the AUGAGAUAA minigene expression together with low level of Pth activity (pth(rap)) to deplete tRNA ${ }^{\text {Arg }}{ }^{44}$ In the present studies, we assign a second direct role to Pth as a factor that rescues stalled ribosomes with oligopep-tRNA as shown in pathway (i). This new concept was created because one of the systems we used did not require the expression of the minigene AUGAGAUAA for induction of stalled ribosomes ${ }^{56}$ (Fig. 1). In this system, when the level of any of the factors tested, including Pth, was reduced or deleted, stalling occurred (Fig. 1b). The accumulated oligopep-tRNA ${ }^{\text {Arg4 }}$ was found on the ribosome (Fig. 2). It is possible that in the absence of Pth, a large amount of free oligopep-tRNA ${ }^{\mathrm{Arg} 4}$ may accumulate in the supernatant. Therefore, one can argue that this oligopep-tRNA ${ }^{\mathrm{Arg} 4}$ may bind back to the ribosome. This possibility, however, is unlikely because RRF and EF-G are known to release mRNA actively. ${ }^{11,74}$ Since RRF is present in the Pth(rap) strain, there would be no ribosome with mRNA only for the free pep-tRNA ${ }^{A r g} 4$ binds to. Regarding the Pth activity on the ribosome-bound pep-tRNA, it is known that Pth cleaves about $30 \%$ of the ester bonds of ribosome-bound $\mathrm{N}$-acetyl-alanyl-phenylalanyl-tRNA ${ }^{75}$ and the ribosome's protection of pep-tRNA from Pth is not $100 \% .{ }^{42}$ The new role of Pth, hydrolysis of ribosome-bound oligopep-tRNA, does not work for hydrolyzing ribosome-bound incomplete polypep-tRNA. Thus, in the experiments described in Figs. 4 and 5, the pth(rap) alone did not cause ribosome stalling at AGAAGA codons corresponding to 
the $352^{\text {nd }}$ and $353^{\text {rd }}$ positions. For stalling before these codons to occur, we needed another mutation such as frr(Ts) (RRFTs) (Figs. 4 and 5) or prfA1(Ts) (RF1Ts) (Fig. 6c) in addition to $p t h(r a p)$. Clearly, Pth is playing the role of depleting tRNA ${ }^{A r g 4}$ together with the minigene placed in the plasmid (Fig. 4a). We presume that Pth cannot hydrolyze ribosome-bound polypep-tRNA ${ }^{\text {leu1 }}$ because the nascent polypeptide bound to tRNA leu1 is fully secured in the $50 \mathrm{~S}$ tunnel, while the ester-bond of oligopep-tRNA ${ }^{\mathrm{Arg} 4}$ is more exposed to the solvent and may be more accessible to Pth.

\section{ACKNOWLEDGMENTS}

This research was supported by CBRI (to HK and AK) and by grant No. 54206 of CONACYT, Mexico, to GG.). S.V.-D was supported by AK and HK. We thank the following individuals who gave us strains or constructs used in this work: Drs. L. A. Isaksson and R. Buckingham for US486; Y. Nakamura and K. Ito for YN2971, Carol Gross, for CAG12025; C. Yanofsky and R. Cruz-Vera for SVS1144, R. T. Sauer for W3110 ssrA::Kan and B. J. Jr. Del Tito for pDC952.

\section{FIGURE LEGENDS}

Figure 1. Oligopep-tRNA ${ }^{\text {Arg4 }}$ accumulates upon induction of the lacZ AGA3-6 mRNA in strains defective or depletion in one of the following: Pth (pth(rap)), RRF (frrTs), RF3 ( $\Delta$ prfC), and tmRNA $(\Delta s s r A)$. (a) placZ AGA3-6 construct. The boxed sequences represent ORFs. Numbers under boxes show positions of AGAs and termination codon. Italics indicate the EcoRI and Hindlll sites used in the construction. The placZ AGA3-6 construct is under the control of $\mathrm{P}_{\text {tac }} / \mathrm{O}_{\text {lac }}$ promoter-operator and $\mathrm{T}_{\text {rrnb }}$ is the 
transcriptional terminator. The Shine-Dalgarno sequence is underlined. (b) Northern blot of tRNA ${ }^{\text {Arg4 }}$ and oligopep-tRNA ${ }^{\text {Arg4 }}$. Cells harboring the placZ AGA3-6 construct (left panel) or cells co-transformed with pDC952 expressing tRNA ${ }^{\text {Arg4 }}$ (right panel) were grown at $32^{\circ} \mathrm{C}$ to $0.3 \mathrm{OD}_{600}$. Cultures were shifted up to $42^{\circ} \mathrm{C}$ (non-permissive temperature for frrTs) for $5 \mathrm{~min}$. Then, lacZ AGA3-6 mRNA expression was induced with $1 \mathrm{mM}$ IPTG for $10 \mathrm{~min}$ at $42^{\circ} \mathrm{C}$. Oligopep-tRNA ${ }^{\mathrm{Arg} 4}$ and $\mathrm{tRNA}^{\mathrm{Arg} 4}$ were isolated and detected by Northern blotting as described in Materials and Methods. Positions of free tRNA $^{\text {Arg4 }}$ and oligopep-tRNA ${ }^{\text {Arg4 }}$ are indicated on the left of the panel. The lane numbers are shown on the gel. The numbers below each lane represent percentage of oligopep-tRNA ${ }^{\text {Arg4 }}$ of total tRNA ${ }^{\text {Arg4 }}$.

Figure 2. Oligopep-tRNA ${ }^{\mathrm{Arg} 4}$ and $5^{\prime}$-end lacZ AGA3-6 mRNA fragments are associated with stalled ribosomes in strains defective or depletion in one of the following: Pth (pth(rap)), RRF (frrTs), RF3 ( $\Delta p r f C)$, and tmRNA $(\Delta s s r A)$. Cells transformed with placZ AGA3-6 construct were grown and induced as described in the legend to Fig. 1. S-30 was prepared and treated with (+) or without (-) puromycin. S-100 (S) and ribosomal (R) fractions were analyzed for the presence of oligopep-tRNA ${ }^{\operatorname{Arg} 4}$ (a) or lacZ AGA3-6 mRNA (b) by Northern blotting using specific $\left[{ }^{32} \mathrm{P}\right]$-oligonucleotides (Material and Methods, Table 2). The loading controls showing ribosomal RNA after ethidium bromide staining are indicated in the lower panel. Positions of various molecular weight RNAs are indicated on the right side of the panels. 
Figure 3. Pth, RF3, and RRF but not tmRNA rescue ribosomes stalled at UAA of minigene AUGAGAUAA. (a) pAGA minigene construct placed in various strains indicated above the lanes of Northern blot analysis (b). Plasmid features were as described in Fig. 1a. (b and c) Northern blot analysis showing Met-Arg-tRNA ${ }^{\operatorname{Arg} 4}$. AGA minigenes were introduced into strains indicated above each lane and induced as described in Fig. 1. (b) Total RNA obtained and treated with $\mathrm{CuSO}_{4}$ as in Fig. 1, (c) Ribosome and supernatant fractions obtained as indicated in Fig. 2 and these fractions were treated with (+) or without (-) $\mathrm{CuSO}_{4}$.

Figure 4. Reduction of RRF activity, deleting RF3 or tmRNA promote the stalling of ribosomes at the middle of lacZ AGA352-353 mRNA: Accumulation of truncated $\beta$-galpolypeptide. (a) pHZAGA352-353-MR construct. This construct encodes an N-terminal His-6 tagged lacZ variant carrying two AGA codons at positions 352 and 353 and a minigene AUGAGAUAA in the same operon. (b) Western blots showing complete and incomplete $\beta$-gal-polypeptides. Where indicated as 32/42, induction was performed for 5 min at $32^{\circ} \mathrm{C}$ to allow sequestering of tRNA ${ }^{\text {Arg4 }}$ by the AGA minigene expression. Cells were then switched to $42^{\circ} \mathrm{C}$ for 15 min to inactivate temperature-sensitive RRF. All other cells were grown and induced at 28 or $32^{\circ} \mathrm{C}$ and no temperature shift was performed. Lane 1 shows a truncated $\beta$-gal polypeptide generated by expressing a lac $Z$ variant carrying a stop codon at position 354 (Table 1). In lane 8, the frrTs cells were cotransformed with pPEN907 (plasmid harboring wild type frr expressing RRF constitutively, Table 1). Arrow 1, complete $\beta$-gal; arrow 2, truncated $\beta$-gal; arrow 3, 
intermediate size $\beta$-gal bands that correspond to fragments normally produced by high rate translation of lacZ.

Figure 5. The incomplete $\beta$-gal-polypeptide is associated with the stalled ribosome as polypep-tRNA ${ }^{\text {Leu1 }}$ in double mutants of $p$ th(rap) with temperature sensitive RRF or missing RF3. P90C mutants harboring the plasmid pHZ352-353-MR were induced as described in Fig. 4 (32/42) and subjected to SDGC. (a) Ribosome profiles in SDGC. The numbers above the plots indicate the gradient fractions used for Tris-acetate SDSPAGE. (b) Ribosome-bound incomplete $\beta$-gal-polypeptide (arrows 2 and 3 ) detected by Western blotting using anti-His-6 antibody. Numbers above each lane of the gel correspond to fraction numbers shown in panel A. (c) Ribosome-bound incomplete $\beta$ gal-polypep-tRNA ${ }^{\text {Leu1 }}$ (arrow 2) detected by Northern blot for tRNA ${ }^{\text {Leu1 }}$. Strains are indicated above panels $\mathrm{A}, \mathrm{B}$, and $\mathrm{C}$. For panel $\mathrm{Cc}$, a separate gradient from panels $\mathrm{Ca}$ and $\mathrm{Cb}$ was run. Therefore, the OD profile of the SDGC (Ca) does not correspond to fraction numbers (indicated above each lane in panel Cc). Molecular weight markers are shown on the right margins in (b) and (c). The white arrows show the ribosomal peak. Arrow 1, complete $\beta$-gal; arrow 2, incomplete $\beta$-gal-polypep-tRNA ${ }^{\text {Leu1}}$; arrow 3 , incomplete $\beta$-gal polypeptide; arrow 4 , free tRNA ${ }^{\text {Leu1 }}$. Transfer RNA ${ }^{\text {Leu1 }}$ can be seen as the tail toward the ribosomal fractions because fractions were collected from the top and some of tRNA ${ }^{\text {Leu1 }}$ contaminate the ribosomal fractions.

Figure 6. Loss of RF1 or RF2 results in accumulation of oligo- and incomplete $\beta$-galpolypep-tRNAs. (a) Northern blot showing oligopep-tRNA ${ }^{\text {Arg } 4}$ and tRNA ${ }^{\text {Arg4 }}$ in US486 
(prfA1Ts, temperature-sensitive RF1). Cells harboring placZ AGA3-6 construct (Fig. 1a) were grown at $32^{\circ} \mathrm{C}$, treated with $1 \mathrm{mM} \mathrm{IPTG}$ for 2 min (lane 2) or not (lane 1). For lanes 3 and 4 they were identically treated as lane 2 except that temperature was raised as indicated followed by the addition of IPTG. (b) As in (a) with YN2971 (prfB2Ts, temperature-sensitive RF2). (c) Western blot showing incomplete $\beta$-gal-polypeptide upon loss of RF1. Mutants P90C prfA1Ts (lane 1) and P90C pth(rap)/prfA1Ts (lanes 24) transformed with pHZ352-353-MR were grown and the plasmid expression was induced at $32^{\circ} \mathrm{C}$ and shifted to $42^{\circ} \mathrm{C}$ the analysis was carried out as in the Materials and Methods. Where indicated, the extract was treated with (lanes 3 and 4) or without (lanes 1 and 2) Pth. Molecular weight markers are shown in the right margin. Arrow 1, complete $\beta$-gal; arrow 2 , incomplete $\beta$-gal-polypep-tRNA ${ }^{\text {Leu1; }}$; arrow 3 , incomplete $\beta$-galpolypeptide.

Figure 7. Model for the possible roles of factors in the rescue of stalled ribosomes. (Complex A), stalled ribosomes with pep-tRNA at the P-site, deacylated tRNA at the Esite, and empty A-site. In pathway (i) Pep-tRNA at the P-site is hydrolyzed by the release factors or by Pth. Complex (B), Ribosomes with mRNA and tRNA at the P-site. The structure is similar to the PoTC. This is disassembled to (E), ribosomal subunits, released tRNA and mRNA. Complex $(B)$ to $(E)$ is carried out by the recycling system involving RRF and EF-G. Pathway (ii) should work for all stalled ribosomes except for those with the termination triplet at the A-site. Complex (A) loses the 3' portion of mRNA by the action of RelE (a specific ribonuclease for the A-site bound mRNA) to become complex (C), which does not have the 3' portion of the bound mRNA. Transfer mRNA 
binds to this and the ribosome reaches the termination codon of tmRNA followed by the conventional recycling by RRF and EF-G. In pathway (iii), "drop off" of pep-tRNA from the ribosome by RRF, RF3 and EF-G leads to the hypothetical intermediate (D), which is then disassembled by RRF and EF-G. The "drop off" rates of pep-tRNA decrease as the size of peptide chain increases. ${ }^{76,77}$ Complex (D) may not exist and the release of deacylated tRNA at the E-site may take place simultaneously with the release of peptRNA.

\section{REFERENCES}

1. Capecchi, M. R. (1967). Polypeptide chain termination in vitro: isolation of a release factor. Proc Natl Acad Sci U S A 58, 1144-1151.

2. Scolnick, E., Tompkins, R., Caskey, T. \& Nirenberg, M. (1968). Release factors differing in specificity for terminator codons. Proc Natl Acad Sci U S A 61, 768774.

3. Caskey, C. T., Tompkins, R., Scolnick, E., Caryk, T. \& Nierenberg, M. (1968). Sequential translation of trinucleotide codons for initiation and termination of protein synthesis. Science 162, 135-138.

4. Petry, S., Brodersen, D. E., Murphy, F. V. t., Dunham, C. M., Selmer, M., Tarry, M. J., Kelley, A. C. \& Ramakrishnan, V. (2005). Crystal structures of the ribosome in complex with release factors RF1 and RF2 bound to a cognate stop codon. Cell 123, 1255-66.

5. Laurberg, M., Asahara, H., Korostelev, A., Zhu, J., Trakhanov, S. \& Noller, H. F. (2008). Structural basis for translation termination on the $70 \mathrm{~S}$ ribosome. Nature 454, 852-857.

6. Petry, S., Weixlbaumer, A. \& Ramakrishnan, V. (2008). The termination of translation. Curr Opin Struct Biol 18, 70-7.

7. Freistroffer, D. V., Pavlov, M. Y., MacDougall, J., Buckingham, R. H. \& Ehrenberg, M. (1997). Release factor RF3 in E.coli accelerates the dissociation of release factors RF1 and RF2 from the ribosome in a GTP-dependent manner. EMBO J 16, 4126-4133.

8. Grentzmann, G., Kelly, P. J., Laalami, S., Shuda, M., Firpo, M. A., Cenatiempo, Y. \& Kaji, A. (1998). Release factor RF-3 GTPase activity acts in disassembly of the ribosome termination complex. Rna 4, 973-83. 
9. Hirashima, A. \& Kaji, A. (1972). Purification and properties of ribosome-releasing factor. Biochemistry 11, 4037-4044.

10. Karimi, R., Pavlov, M. Y., Buckingham, R. H. \& Ehrenberg, M. (1999). Novel roles for classical factors at the interface between translation termination and initiation. Mol Cell 3, 601-609.

11. Hirokawa, G., Demeshkina, N., Iwakura, N., Kaji, H. \& Kaji, A. (2006). The ribosome-recycling step: consensus or controversy? Trends Biochem Sci 31, 143-149.

12. Pai, R. D., Zhang, W., Schuwirth, B. S., Hirokawa, G., Kaji, H., Kaji, A. \& Cate, J. $H$. (2008). Structural insights into ribosome recycling factor interactions with the 70 S ribosome. J Mol Biol 376, 1334-47.

13. Weixlbaumer, A., Petry, S., Dunham, C. M., Selmer, M., Kelley, A. C. \& Ramakrishnan, V. (2007). Crystal structure of the ribosome recycling factor bound to the ribosome. Nat Struct Mol Biol 14, 733-7.

14. Dunkle, J. A., Xiong, L., Mankin, A. S. \& Cate, J. H. (2010). Structures of the Escherichia coli ribosome with antibiotics bound near the peptidyl transferase center explain spectra of drug action. Proc Natl Acad Sci U S A.

15. Gryczan, T. J., Grandi, G., Hahn, J., Grandi, R. \& Dubnau, D. (1980). Conformational alteration of mRNA structure and the posttranscriptional regulation of erythromycin-induced drug resistance. Nucleic Acids Res 8, 608197.

16. Horinouchi, S. \& Weisblum, B. (1980). Posttranscriptional modification of mRNA conformation: Mechanism that regulates erythromycin-induced resistance. Proc Natl Acad Sci U S A 77, 7079-7083.

17. Craigen, W. J. \& Caskey, C. T. (1986). Expression of peptide chain release factor 2 requires high-efficiency frameshift. Nature 322, 273-275.

18. McNicholas, P., Salavati, R. \& Oliver, D. (1997). Dual regulation of Escherichia coli secA translation by distinct upstream elements. J Mol Biol 265, 128-41.

19. Gong, F., Ito, K., Nakamura, Y. \& Yanofsky, C. (2001). The mechanism of tryptophan induction of tryptophanase operon expression: Tryptophan inhibits release factor-mediated cleavage of TnaC-peptidyl-tRNAPro. Proc Natl Acad Sci U S A 98, 8997-9001.

20. Gong, F. \& Yanofsky, C. (2002). Instruction of translating ribosome by nascent peptide. Science 297, 1864-7.

21. Murakami, A., Nakatogawa, H. \& Ito, K. (2004). Translation arrest of SecM is essential for the basal and regulated expression of SecA. Proc Natl Acad Sci U S A 101, 12330-12335.

22. Gong, M., Cruz-Vera, L. R. \& Yanofsky, C. (2007). Ribosome Recycling Factor and Release Factor 3 Action Promotes TnaC-Peptidyl-tRNA Dropoff and Relieves Ribosome Stalling during Tryptophan Induction of tna Operon Expression in Escherichia coli. J Bacteriol 189, 3147-3155.

23. Vazquez-Laslop, N., Thum, C. \& Mankin, A. S. (2008). Molecular mechanism of drug-dependent ribosome stalling. Mol Cell 30, 190-202.

24. Cruz-Vera, L. R., Yang, R. \& Yanofsky, C. (2009). Tryptophan Inhibits Proteus vulgaris TnaC Leader Peptide Elongation, Activating tna Operon Expression. $J$ Bacteriol 191, 7001-7006. 
25. Shirole, N., Balasubramanian, S., Yanofsky, C. \& Cruz-Vera, L. (2011). Isolation of Translating Ribosomes Containing Peptidyl-tRNAs for Functional and Structural Analyses. J Vis Exp.

26. Janssen, B. D. \& Hayes, C. S. (2009). Kinetics of paused ribosome recycling in Escherichia coli. J Mol Biol 394, 251-267.

27. Handa, Y., Inaho, N. \& Nameki, N. (2011). YaeJ is a novel ribosome-associated protein in Escherichia coli that can hydrolyze peptidyl-tRNA on stalled ribosomes. Nucleic Acids Res 39, 1739-1748.

28. Chadani, Y., Ono, K., Kutsukake, K. \& Abo, T. (2011). Escherichia coli YaeJ protein mediates a novel ribosome-rescue pathway distinct from SsrA- and ArfAmediated pathways. Mol Microbiol.

29. Chadani, Y., Matsumoto, E., Aso, H., Wada, T., Kutsukake, K., Sutou, S. \& Abo, T. (2011). trans-translation-mediated tight regulation of the expression of the alternative ribosome-rescue factor ArfA in Escherichia coli. Genes Genet Syst 86, 151-63.

30. Gallant, J. A. \& Lindsley, D. (1998). Ribosomes can slide over and beyond "hungry" codons, resuming protein chain elongation many nucleotides downstream. Proc Natl Acad Sci U S A 95, 13771-13776.

31. Keiler, K. C., Waller, P. R. H. \& Sauer, R. T. (1996). Role of a peptide tagging system in degradation of proteins synthesized from damaged messenger RNA. Science 271, 990-993.

32. Roche, E. D. \& Sauer, R. T. (1999). SsrA-mediated peptide tagging caused by rare codons and tRNA scarcity. EMBO J 18, 4579-4589.

33. Pedersen, K., Zavialov, A. V., Pavlov, M. Y., Elf, J., Gerdes, K. \& Ehrenberg, M. (2003). The Bacterial Toxin RelE Displays Codon-Specific Cleavage of mRNAs in the Ribosomal A Site. Cell 112, 131-140.

34. Hayes, C. S. \& Sauer, R. T. (2003). Cleavage of the A site mRNA codon during ribosome pausing provides a mechanism for translational quality control. $\mathrm{Mol}$ Cell 12, 903-11.

35. Asano, K., Kurita, D., Takada, K., Konno, T., Muto, A. \& Himeno, H. (2005). Competition between trans-translation and termination or elongation of translation. Nucleic Acids Res 33, 5544-52.

36. Li, X., Yagi, M., Morita, T. \& Aiba, H. (2008). Cleavage of mRNAs and role of tmRNA system under amino acid starvation in Escherichia coli. Mol Microbiol 68, 462-73.

37. Heurgue-Hamard, V., Karimi, R., Mora, L., MacDougall, J., Leboeuf, C., Grentzmann, G., Ehrenberg, M. \& Buckingham, R. H. (1998). Ribosome release factor RF4 and termination factor RF3 are involved in dissociation of peptidyltRNA from the ribosome. Embo J 17, 808-816.

38. Watanabe, Y., Nakamura, Y. \& Ito, K. (2010). A novel class of bacterial translation factor RF3 mutations suggests specific structural domains for premature peptidyl-tRNA drop-off. FEBS Lett 584, 790-4.

39. Rao, A. R. \& Varshney, U. (2001). Specific interaction between the ribosome recycling factor and the elongation factor $\mathrm{G}$ from Mycobacterium tuberculosis mediates peptidyl-tRNA release and ribosome recycling in Escherichia coli. $E M B O ~ J ~ 20,2977-2986$. 
40. Singh, N. S., Das, G., Seshadri, A., Sangeetha, R. \& Varshney, U. (2005). Evidence for a role of initiation factor 3 in recycling of ribosomal complexes stalled on mRNAs in Escherichia coli. Nucleic Acids Res 33, 5591-601.

41. Singh, N. S., Ahmad, R., Sangeetha, R. \& Varshney, U. (2008). Recycling of ribosomal complexes stalled at the step of elongation in Escherichia coli. $\mathrm{J} \mathrm{Mol}$ Biol 380, 451-464.

42. Menninger, J. R., Mulholland, M. C. \& Stirewalt, W. S. (1970). Peptidyl-tRNA hydrolase and protein chain termination. Biochim Biophys Acta 217, 496-511.

43. Chapeville, F., Yot, P. \& Paulin, D. (1969). Enzymatic hydrolysis of N-acylaminoacyl transfer RNAs. Cold Spring Harb Symp Quant Biol 34, 493-498.

44. Hernandez-Sanchez, J., Valadez, J. G., Herrera, J. V., Ontiveros, C. \& Guarneros, G. (1998). lambda bar minigene-mediated inhibition of protein synthesis involves accumulation of peptidyl-tRNA and starvation for tRNA. EMBO J 17, 3758-65.

45. Cruz-Vera, L. R., Hernandez-Ramon, E., Perez-Zamorano, B. \& Guarneros, G. (2003). The Rate of Peptidyl-tRNA Dissociation from the Ribosome during Minigene Expression Depends on the Nature of the Last Decoding Interaction. $J$ Biol Chem 278, 26065-26070.

46. Henderson, D. \& Weil, J. (1976). A mutant of Escherichia coli that prevents growth of phage lambda and is bypassed by lambda mutants in a nonessential region of the genome. Virology 71, 546-59.

47. Tenson, T., Herrera, J. V., Kloss, P., Guarneros, G. \& Mankin, A. S. (1999). Inhibition of translation and cell growth by minigene expression. J Bacteriol 181, 1617-22.

48. Varshney, U., Lee, C. P. \& RajBhandary, U. L. (1991). Direct analysis of aminoacylation levels of tRNAs in vivo. Application to studying recognition of Escherichia coli initiator tRNA mutants by glutaminyl-tRNA synthetase. $\mathrm{J}$ Biol Chem 266, 24712-24718.

49. Vivanco-Dominguez, S., Cruz-Vera, L. R. \& Guarneros, G. (2006). Excess of charged tRNA ${ }^{\text {Lys }}$ maintains low levels of peptidyl-tRNA hydrolase in pth(Ts) mutants at a non-permissive temperature. Nucleic Acids Res 34, 1564-1570.

50. Schofield, P. \& Zamecnik, P. C. (1968). Cupric ion catalysis in hydrolysis of aminoacyl-tRNA. Biochim Biophys Acta 155, 410-6.

51. Cruz-Vera, L. R., Magos-Castro, M. A., Zamora-Romo, E. \& Guarneros, G. (2004). Ribosome stalling and peptidyl-tRNA drop-off during translational delay at AGA codons. Nucleic Acids Res 32, 4462-4468.

52. Sambrook, J., Fritsch, E. F. \& Maniatis, T. (1989). Molecular Cloning: $A$ Laboratory Manual. 2nd edit, Cold Spring Harbor Laboratory Press, Cold Spring Harbor, NY.

53. Laemmli, U. K. (1970). Cleavage of structural proteins during the assembly of the head of bacteriophage T4. Nature 227, 680-685.

54. Moll, I., Hirokawa, G., Kiel, M. C., Kaji, A. \& Blasi, U. (2004). Translation initiation with 70 ribosomes: an alternative pathway for leaderless mRNAs. Nucleic Acids Res 32, 3354-3363. 
55. Kirchdoerfer, R. N., Huang, J. J., Isola, M. K. \& Cavagnero, S. (2007).

Fluorescence-based analysis of aminoacyl- and peptidyl-tRNA by low-pH sodium dodecyl sulfate-polyacrylamide gel electrophoresis. Anal Biochem 364, 92-94.

56. Zamora-Romo, E., Cruz-Vera, L. R., Vivanco-Dominguez, S., Magos-Castro, M. A. \& Guarneros, G. (2007). Efficient expression of gene variants that harbour AGA codons next to the initiation codon. Nucleic Acids Res 35, 5966-5974.

57. Garcia-Villegas, M. R., De La Vega, M., Galindo, J. M., Segura, M., Buckingham, R. H. \& Guarneros, G. (1991). Peptidyl-tRNA hydrolase is involved in lambda inhibition of host protein synthesis. EMBO J 10, 3549-3555.

58. Cruz-Vera, L. R., Toledo, I., Hernandez-Sanchez, J. \& Guarneros, G. (2000). Molecular Basis for the Temperature Sensitivity of Escherichia coli pth(Ts). J Bacteriol 182, 1523-1528.

59. Janosi, L., Mottagui-Tabar, S., Isaksson, L. A., Sekine, Y., Ohtsubo, E., Zhang, S., Goon, S., Nelken, S., Shuda, M. \& Kaji, A. (1998). Evidence for in vivo ribosome recycling, the fourth step in protein biosynthesis. EMBO J 17, 11411151.

60. Kurata, S., Weixlbaumer, A., Ohtsuki, T., Shimazaki, T., Wada, T., Kirino, Y., Takai, K., Watanabe, K., Ramakrishnan, V. \& Suzuki, T. (2008). Modified uridines with C5-methylene substituents at the first position of the tRNA anticodon stabilize U.G wobble pairing during decoding. J Biol Chem 283, 1880111.

61. Geigenmuller, U., Hausner, T. P. \& Nierhaus, K. H. (1986). Analysis of the puromycin reaction. The ribosomal exclusion principle for AcPhe-tRNA binding re-examined. Eur J Biochem 161, 715-21.

62. Baker, K. E. \& Mackie, G. A. (2003). Ectopic RNase E sites promote bypass of 5'-end-dependent mRNA decay in Escherichia coli. Mol Microbiol 47, 75-88.

63. Steitz, J. A. (1969). Polypeptide Chain Initiation: Nucleotide Sequences of the Three Ribosomal Binding Sites in Bacteriophage R17 RNA. Nature 224, 957-964.

64. Delgado-Olivares, L., Zamora-Romo, E., Guarneros, G. \& Hernandez-Sanchez, J. (2006). Codon-specific and general inhibition of protein synthesis by the tRNAsequestering minigenes. Biochimie 88, 793-800.

65. Manley, J. L. (1978). Synthesis and degradation of termination and prematuretermination fragments of beta-galactosidase in vitro and in vivo. $J$ Mol Biol 125, 407-32.

66. Seidelt, B., Innis, C. A., Wilson, D. N., Gartmann, M., Armache, J. P., Villa, E., Trabuco, L. G., Becker, T., Mielke, T., Schulten, K., Steitz, T. A. \& Beckmann, R. (2009). Structural insight into nascent polypeptide chain-mediated translational stalling. Science 326, 1412-5.

67. Zaher, H. S. \& Green, R. (2009b). Quality control by the ribosome following peptide bond formation. Nature 457, 161-166.

68. Hirashima, A. \& Kaji, A. (1973). Role of elongation factor $G$ and a protein factor on the release of ribosomes from messenger ribonucleic acid. $J$ Biol Chem 248, 7580-7587.

69. Frolova, L., LeGoff, X., Zhouravleva, G., Davydova, E., Philippe, M. \& Kisselev, L. (1996). Eukaryotic polypeptide chain release factor eRF3 is an eRF1- and ribosome-dependent guanosine triphosphatase. RNA 2, 334-341. 
70. Ishida, H. \& Hayward, S. (2008). Path of nascent polypeptide in exit tunnel revealed by molecular dynamics simulation of ribosome. Biophys J 95, 59625973.

71. Sohmen, D., Harms, J. M., Schlunzen, F. \& Wilson, D. N. (2009). Enhanced SnapShot: Antibiotic Inhibition of Protein Synthesis II. Cell 139, 212-212 e1.

72. Gillet, R. \& Felden, B. (2001). Emerging views on tmRNA-mediated protein tagging and ribosome rescue. Mol Microbiol 42, 879-85.

73. Ivanova, N., Pavlov, M. Y., Felden, B. \& Ehrenberg, M. (2004). Ribosome rescue by tmRNA requires truncated mRNAs. $J$ Mol Biol 338, 33-41.

74. Ogawa, K. \& Kaji, A. (1975). Requirement for ribosome-releasing factor for the release of ribosomes at the termination codon. Eur J Biochem 58, 411-419.

75. Vogel, Z., Vogel, T., Zamir, A. \& Elson, D. (1971). The Protection by $70 \mathrm{~S}$ Ribosomes of N-Acyl-aminoacyl-tRNA against Cleavage by Peptidyl-tRNA Hydrolase and its Use to Assay Ribosomal Association. Eur J Biochem 21, 582592.

76. Heurgue-Hamard, V., Dincbas, V., Buckingham, R. H. \& Ehrenberg, M. (2000). Origins of minigene-dependent growth inhibition in bacterial cells. Embo J 19, 2701-2709.

77. Jacinto-Loeza, E., Vivanco-Dominguez, S., Guarneros, G. \& Hernandez-Sanchez, J. (2008). Minigene-like inhibition of protein synthesis mediated by hungry codons near the start codon. Nucleic Acids Res 36, 4233-41.

78. Singer, M., Baker, T. A., Schnitzler, G., Deischel, S. M., Goel, M., Dove, W., Jaacks, K. J., Grossman, A. D., Erickson, J. W. \& Gross, C. A. (1989). A collection of strains containing genetically linked alternating antibiotic resistance elements for genetic mapping of Escherichia coli. Microbiol Rev 53, 1-24.

79. Yanofsky, C., Horn, V. \& Nakamura, Y. (1996). Loss or overproduction of polypeptide release factor 3 influences expression of the tryptophanase operon of Escherichia coli. J Bacteriol 178, 3755-3762.

80. Mora, L., Zavialov, A., Ehrenberg, M. \& Buckingham, R. H. (2003). Stop codon recognition and interactions with peptide release factor RF3 of truncated and chimeric RF1 and RF2 from Escherichia coli. Mol Microbiol 50, 1467-76.

81. Coulondre, C. \& Miller, J. H. (1977). Genetic Studies of the lac Repressor: III. Additional Correlation of Mutational Sites with Specific Amino Acid Residues. J Mol Biol 117, 525-575.

82. Miller, J. H. (1992). A Short Course in Bacterial Genetics: A Laboratory Manual and Handbook for Escheichia coli and Related Bacteria. Illustrated edit (Miller, J. H., Ed.), 1, Cold Spring Harbor Laboratory Press, Cold Spring Harbor, NY.

83. Kawakami, K., Inada, T. \& Nakamura, Y. (1988). Conditionally lethal and recessive UGA-suppressor mutations in the prfB gene encoding peptide chain release factor 2 of Escherichia coli. J Bacteriol 170, 5378-5381.

84. Del Tito, B. J., Jr., Ward, J. M., Hodgson, J., Gershater, C. J., Edwards, H., Wysocki, L. A., Watson, F. A., Sathe, G. \& Kane, J. F. (1995). Effects of a minor isoleucyl tRNA on heterologous protein translation in Escherichia coli. J Bacteriol 177, 7086-91.

85. Emilsson, V., Naslund, A. K. \& Kurland, C. G. (1993). Growth-rate-dependent accumulation of twelve tRNA species in Escherichia coli. J Mol Biol 230, 483-91. 
86. Emilsson, V. \& Kurland, C. G. (1990). Growth rate dependence of transfer RNA abundance in Escherichia coli. Embo J 9, 4359-66. 